\title{
Cochlear implants: the hazards of unexpected success
}

\author{
Jay T. Rubinstein MD PhD
}

$\mathrm{T}$ hirty years ago, cochlear implants were in their infancy as research projects conducted at a handful of institutions around the world. The devices were crude, typically manufactured in a university or other noncommercial engineering laboratory, unapproved by any government regulatory agency, and implanted in experimental animals or humans for short periods. There was substantial skepticism, not only about the efficacy of the existing devices but also about the entire concept. It simply was not believable to many that such a device could ever functionally replace the complex and elegant biophysics of the cochlea. Pioneering implant surgeons were accused of performing unethical human studies. I was strongly encouraged, as were other interested students, to seek alternative, and putatively more rewarding, dissertation projects.

Today, cochlear implants are a global commercial enterprise, with three major manufacturers and a few smaller entities developing or marketing these devices. Various implants are approved by government regulatory agencies across the developed world and are reimbursed to various degrees by commercial and government payors. Financial analysts now pay as much attention to the results of clinical trials of these devices as do clinicians. It is a new world for these remarkable devices because they represent a highly effective and cost-effective treatment $^{1}$ of severe-profound sensorineural hearing loss in both children and adults.

It is now well established that prelingually deafened children (loss of hearing before speech begins) can develop near-normal levels of speech and language and achieve normal educational milestones when given an implant at an early age. ${ }^{2}$ Postlingually deaf adults, even octogenarians, ${ }^{3}$ can usually speak on the telephone and, in some cases, achieve normal levels of speech understanding in quiet environments within a year of implantation. This level of success is beyond the wildest dreams of the pioneering engineers and clinicians. So what are the hazards?

Implants previously underpromised and over delivered. In an era in which patients were unable to understand speech with a hearing aid, any chance of improvement was welcome. Patients were warned that they might not benefit from an implant but would often proceed anyway because there was little risk associated with implanting a device in an ear that already had essentially no hearing. Even minimal postoperative speech perception would represent a substantial auditory benefit. As the devices improved over time, so did the risk-benefit ratio, at least for people with severe or total hearing loss.

Today there are several quite promising nontraditional applications of implants that challenge this highly favourable risk-benefit analysis because they either involve implanting a device in a partly hearing ear or demand extremely high levels of performance from an ear with an implanted device (implanted ear) to provide benefit.

Because speech discrimination with implants has improved dramatically, the criteria for implantation has appropriately broadened to include patients with significant levels of residual hearing. Although residual speech discrimination is a positive predictor of outcome, it is a weak one. ${ }^{4}$ Therefore, even if speech outcomes were the only concern, there is certainly a limit to the level of residual hearing beyond which implantation is likely to do more harm than good. Hence, carefully controlled studies must be performed before advocating widespread exceptions to existing preoperative criteria. Given the known superiority of even low levels of acoustic hearing for the perception of musical pitch and timbre in comparison to the typically poor performance of implants in this important domain, ${ }^{5}$ such studies may require the use of both music and speech perception in noise as outcome measures.

Electroacoustic or hybrid cochlear implants attempt to preserve low-frequency acoustic sensi-
Competing interests: Jay Rubinstein has served as a paid consultant and received research funding from Advanced Bionics Corporation and Cochlear, Ltd., two manufacturers of cochlear implant devices.

This article has been peer reviewed.

Correspondence to: Jay T. Rubinstein, rubinj@u.washington.edu

CMAJ 2012. DOI:10.1503 /cmaj.111743 
tivity in the implanted ear so that the implant provides only the absent high-frequency signals. Current clinical trials of these promising devices involve patients who have normal or near-normal low-frequency hearing and levels of preoperative discrimination exceeding $50 \%$ of monosyllabic words. ${ }^{6}$ The issues being addressed in these trials are whether the outcomes justify the risk of losing residual hearing, or if a standard cochlear implant might result in better overall performance when paired with a hearing aid in the contralateral ear. Early results indicate that the outcomes justify the risks. Complex issues of music and the perception of tonal language (e.g., Mandarin Chinese $)^{7}$ arise here too, as well as the fact that binaural sound localization based on low-frequency interaural timing differences is intact in such patients. This ability would be lost if low-frequency hearing were destroyed, either intentionally with a standard implant or as a complication of hybrid or electroacoustic implantation.

Bilateral cochlear implantation has become commonplace, with the two procedures being performed simultaneously or sequentially. Although there is little doubt that two implants are acoustically superior to one,${ }^{8}$ the cost-efficacy of bilateral implantation is still controversial, ${ }^{1}$ and some people considering a second implant can still use a hearing aid effectively in the unimplanted ear. Such "bimodal hearing" is potentially beneficial for the detection of the pitch of a target speaker's voice, a critical cue for speech perception in fluctuating background noise, ${ }^{9}$ and for semantic and musical pitch perception. Implant performance is still quite limited for such tasks.?

Unilateral, or "single-sided," deafness is another promising application for cochlear implants. ${ }^{10}$ The goals are to restore the benefits of binaural hearing and potentially to suppress tinnitus. Here, the hazard is not losing hearing in the implanted ear. Instead, the risk is that the implant will fail to benefit the patient because of the extraordinary demands being placed on the implanted ear. Achieving binaural hearing benefit from any clinical intervention requires relatively symmetric hearing. ${ }^{11}$ It remains to be seen whether, through careful patient selection (e.g., short duration of deafness), sufficiently high performance can be reliably achieved so that the benefits justify the intervention. The potential for benefit in the domain of tinnitus suppression should also be considere $\mathrm{d}^{12}$ and needs rigorous study.

The extraordinary success of cochlear implants has naturally led to enthusiasm for these newer applications. It is critical as research progresses to remember the limitations of current devices. Their success in enhancing speech discrimination is readily attributable to the robustness of speech and not the temporal or spectral fidelity produced by existing implant technologies. ${ }^{13}$ Limitations of implants in the perception of music, semantic tones and speech in background noise may occur because of their failure to reproduce the spectral detail of the cochlear amplifier, ${ }^{9,14}$ the same reason these devices initially met with such skepticism. Until signal processing, tissue engineering or some mixed technology ${ }^{15}$ allows the restoration of psychoacoustically near-normal hearing with cochlear implants, the adaptation of new applications should be supported by carefully controlled studies. Given the history of these remarkable medical devices, however, it would be unwise to discount that eventual possibility.

\section{References}

1. Bond M, Mealing S, Anderson R, et al. The effectiveness and cost-effectiveness of cochlear implants for severe to profound deafness in children and adults: a systematic review and economic model. Health Technol Assess 2009;13:1-330.

2. Geers AE, Strube MJ, Tobey EA, et al. Epilogue: factors contributing to long-term outcomes of cochlear implantation in early childhood. Ear Hear 2011;32(Suppl 1):84-92.

3. Budenz CL, Cosetti MK, Coelho DH, et al. The effects of cochlear implantation on speech perception in older adults. $J \mathrm{Am}$ Geriatr Soc 2011;59:446-53.

4. Rubinstein JT, Parkinson WS, Tyler RS, et al. Residual speech recognition and cochlear implant performance: effects of implantation criteria. Am J Otol 1999;20:445-52.

5. Gfeller K, Jiang D, Oleson JJ, et al. Temporal stability of music perception and appraisal scores of adult cochlear implant recipients. J Am Acad Audiol 2010;21:28-34.

6. Turner CW, Gantz BJ, Karsten S, et al. Impact of hair cell preservation in cochlear implantation: combined electric and acoustic hearing. Otol Neurotol 2010;31:1227-32.

7. Wang W, Zhou N, Xu L. Musical pitch and lexical tone perception with cochlear implants. Int J Audiol 2011;50:270-8.

8. Basura GJ, Eapen R, Buchman CA. Bilateral cochlear implantation: current concepts, indications, and results. Laryngoscope 2009; 119:2395-401

9. Qin MK, Oxenham AJ. Effects of introducing unprocessed lowfrequency information on the reception of envelope-vocoder processed speech. J Acoust Soc Am 2006;119:2417-26.

10. Arndt S, Aschendorff A, Laszig R, et al. Comparison of pseudobinaural hearing to real binaural hearing rehabilitation after cochlear implantation in patients with unilateral deafness and tinnitus. Otol Neurotol 2011;32:39-47.

11. Yoon YS, Li Y, Kang HY, et al. The relationship between binaural benefit and difference in unilateral speech recognition performance for bilateral cochlear implant users. Int J Audiol 2011;50: 554-65.

12. Van de Heyning P, Vermeire K, Diebl M, et al. Incapacitating unilateral tinnitus in single-sided deafness treated by cochlear implantation. Ann Otol Rhinol Laryngol 2008;117:645-52.

13. Rubinstein JT. How cochlear implants encode speech. Curr Opin Otolaryngol Head Neck Surg 2004;12:444-8.

14. Moore BC. The role of temporal fine structure processing in pitch perception, masking, and speech perception for normalhearing and hearing-impaired people. J Assoc Res Otolaryngol 2008;9:399-406.

15. Wise AK, Fallon JB, Neil AJ, et al. Combining cell-based therapies and neural prostheses to promote neural survival. Neurotherapeutics $2011 ; 8: 774-87$

Affiliation: Jay Rubinstein is with the Departments of Otolaryngology and Bioengineering, Virginia Merrill Bloedel Hearing Research Center, University of Washington, Seattle, Wash.

Acknowledgement: Jay Rubinstein is supported by the National Institutes of Health. 